Received 00th January 20xx, Accepted 00th January 20xx

DOI: $10.1039 / x 0 x \times 00000 x$

www.rsc.org/

\title{
Protein dynamics promote hydride tunnelling in substrate oxidation by aryl-alcohol oxidase $t$
}

\begin{abstract}
Juan Carro, ${ }^{\mathrm{a}}$ Marta Martínez-Júlvez, ${ }^{\mathrm{b}}$ Milagros Medina, ${ }^{\mathrm{b}}$ Angel T. Martínez ${ }^{\mathrm{a}^{*}}$ and Patricia Ferreira ${ }^{\mathrm{b}^{*}}$
The temperature dependence of hydride transfer from the substrate to the N5 of the FAD cofactor during the reductive half-reaction of Pleurotus eryngii aryl-alcohol oxidase (AAO) is assessed here. Kinetic isotope effects on both the presteady state reduction of the enzyme and its steady-state kinetics, with differently deuterated substrates, suggest an environmentally-coupled quantum-mechanical tunnelling process. Moreover, those kinetic data, along with the crystallographic structure of the enzyme in complex with a substrate analogue, indicate that AAO shows a pre-organized active site that would only require the approaching of the hydride donor and acceptor for the tunnelled transfer to take place. Modification of the enzyme's active-site architecture by replacement of Tyr92, a residue establishing hydrophobic interactions with the substrate analogue in the crystal structure, in the $Y 92 \mathrm{~F}, \mathrm{Y} 92 \mathrm{~L}$ and $\mathrm{Y} 92 \mathrm{~W}$ variants resulted in different temperature dependence patterns that indicated a role of this residue in modulating the transfer reaction.
\end{abstract}

\section{Introduction}

Breakage of a $\mathrm{C}-\mathrm{H}$ bond followed by $\mathrm{H}$ transfer is a key biochemical process in which many enzymes rely for their catalysis. ${ }^{1}$ Among them, flavin-containing oxidoreductases of the glucose-methanolcholine oxidase/dehydrogenase (GMC) superfamily are known for their ability to catalyse the oxidative dehydrogenation of a diversity of alcohol compounds. Aryl-alcohol oxidase (AAO) is an extracellular GMC enzyme, widely identified in basidiomycetes genomes, that plays a role in lignocellulose degradation supplying $\mathrm{H}_{2} \mathrm{O}_{2}$ to both ligninolytic peroxidases (in white-rot decay) and Fenton attack to polysaccharides (in brown-rot decay). ${ }^{2-5}$ AAO shows a broad specificity oxidising aromatic lignin-related compounds and fungal metabolites with conjugated primary alcohols. Moreover, they are suitable biocatalysts in the production of bioplastic precursors and in the deracemization of chiral compounds. ${ }^{6,7}$

At present, only the crystal structure of AAO from Pleurotus eryngii is available, ${ }^{8}$ which has been further characterised and used as a model AAO enzyme. ${ }^{9,10}$ This enzyme catalyses the oxidative dehydrogenation of a plethora of aromatic primary alcohols ${ }^{11,12}$ and gem-diols ${ }^{13}$ to their corresponding aldehydes and acids thanks to

\footnotetext{
a. Centro de Investigaciones Biológicas, CSIC, Ramiro de Maeztu 9, E-28040, Madrid, Spain.

${ }^{b .}$ Department of Biochemistry and Molecular and Cellular Biology and Institute for Biocomputation and Physics of Complex Systems, University of Zaragoza, E-50009, Zaragoza, Spain.

†Electronic supplementary information (ESI): Fig. S1 shows the reduction spectra of native AAO and different variants with deuterated alcohol substrate, Fig. S2 shows the $k_{\text {obs }}$ values for the reduction of native AAO and different variants with protiated alcohol substrate, Table S1 provides crystallographic data, Tables S2 and S3 show the effect of temperature on AAO oxidation of protiated and deuterated alcohols (steady-state and transient state constants, respectively), Table S4 shows secondary KIE for AAO reduction with protiated and deuterated alcohols, Tables S5-S7 show the effect of temperature on the transient-state constants for oxidation of protiated and deuterated alcohols by the Y92F, Y92L and Y92W variants, respectively. See DOI: 10.1039/x0xx00000x
}

the (non-synchronous) concerted ${ }^{14}$ : i) abstraction of a $\mathrm{H}^{+}$by the catalytic base His502 conserved in other members of the GMC oxidoreductase superfamily ${ }^{9}$ and ii) stereoselective $\mathrm{H}^{-}$transfer $(\mathrm{HT})$ from the pro- $R$ position of the substrate during the reductive halfreaction of the enzyme (Scheme 1). ${ }^{7,10} \mathrm{H}^{-}$is afterwards transferred to the $\mathrm{N} 5$ of the $\mathrm{FAD}$ cofactor, thereby reducing it, and to $\mathrm{O}_{2}$ during AAO reoxidation. The high primary substrate kinetic isotope effect $(\mathrm{KIE})^{7}$ observed during the reductive half reaction, together with a moderated secondary substrate $\mathrm{KIE}$, suggest hydrogen tunnelling modulation during $\mathrm{HT}^{7}{ }^{7}$ This transfer is the rate-limiting step of the whole AAO catalysis, and its mechanism, the scope of this investigation.

Hydrogen transfer is nowadays regarded as a fully quantummechanical process governed by a hierarchy of enzyme motions. ${ }^{15}$ This "environmentally coupled full tunnelling model" postulates two types of protein motions involved in enzyme catalysis: preorganisation and reorganisation. Pre-organisation involves large portions of protein that could be far from the active site. Reorganisation motions involve heavy atoms within the active site and include "passive" and "active" dynamics. "Passive" dynamics reorganise the active site in a way that create an appropriate ground-state structure for catalysis, while "active" dynamics, also known as gating, help sample donor-acceptor distances (DAD) between substrate and enzyme. Such sampling brings the reacting atoms together so that their wave functions overlap and, thus, particles can be transferred across the barrier width, which is the basic premise of tunnelling. These protein motions have gained momentum because of its involvement in catalysis and, especially, in hydrogen transfer tunnelling. ${ }^{16-20}$

In this work, KIEs on isotopically mono- and di-substituted alcohol substrates, both in steady-state and transient-state kinetics, have been used to assess the temperature dependence of the HT. Then, disruption of the AAO active site by replacement of Tyr92, 

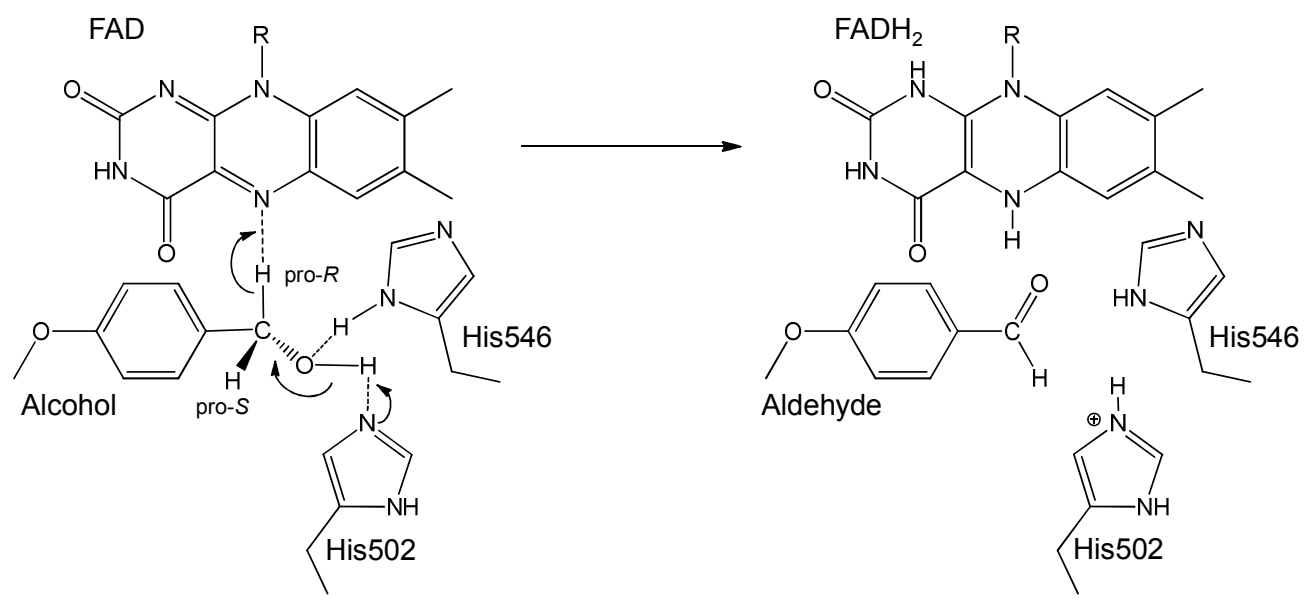

Scheme 1. Scheme of the reductive half-reaction in AAO. The oxidised FAD abstracts a hydride (two electrons plus the pro- $R \mathrm{H}$ from C $\alpha$ position of the alcohol). His502 acts as a catalytic base abstracting a proton from the hydroxyl group of the substrate. His546 contributes to forming a hydrogen bond with the oxygen atom of the hydroxyl group. This reaction gives rise to the oxidised product (an aldehyde), the reduced (hydroquinone) form of FAD, and the double-protonated His502.

involved in the stacking-stabilisation of the substrate at the active site, ${ }^{21}$ has been used to unveil the role that the conformation of the substrate binding pocket plays in promoting the HT. These studies are complemented with crystallographic data that shed light on the catalytically relevant position of the substrate into the active site and the distances over which the $\mathrm{H}^{-}$must be transferred.

\section{Experimental}

\section{Reagents}

Glucose oxidase type VII from Aspergillus niger, glucose and $p$ methoxybenzyl alcohol ( $\geq 98 \%$ ) were purchased from Sigma-Aldrich (Saint Louis, MO, USA). Dideuterated $\left[\alpha-{ }^{2} \mathrm{H}_{2}\right]-p$-methoxybenzyl and monodeuterated $(R)-\left[\alpha-{ }^{2} \mathrm{H}\right]-p$-methoxybenzyl alcohol were synthesised in the Institute of Chemical Synthesis and Homogeneous Catalysis of Zaragoza University (Spain).

\section{Enzyme expression and purification}

Wild-type recombinant (hereinafter native) AAO from Pleurotus eryngii (GenBank accession number F064069) was heterologously produced in Escherichia coli W3110 using the pFLAG1 vector. Y92L, $\mathrm{Y} 92 \mathrm{~F}$ and $\mathrm{Y} 92 \mathrm{~W}$ mutated variants were prepared using the QuikChange ${ }^{\circledast}$ site-directed mutagenesis kit (Stratagene, Santa Clara, CA, USA), as described previously. ${ }^{21}$ The enzyme was overexpressed as inclusion bodies, in vitro refolded in the presence of FAD and purified by ion-exchange chromatography. ${ }^{22}$ Enzyme concentration was determined spectrophotometrically using the molar absorption of native AAO and its $Y 92 \mathrm{~L}, \mathrm{Y} 92 \mathrm{~F}$ and $\mathrm{Y} 92 \mathrm{~W}$ variants $\left(\varepsilon_{463}=11,050 \mathrm{M}^{-1} \cdot \mathrm{cm}^{-1}, \varepsilon_{463}=11,240 \mathrm{M}^{-1} \cdot \mathrm{cm}^{-1}, \varepsilon_{463}=10,044\right.$ $\mathrm{M}^{-1} \cdot \mathrm{cm}^{-1}$ and $\varepsilon_{457}=10,693 \mathrm{M}^{-1} \cdot \mathrm{cm}^{-1}$, respectively). The molar absorption coefficients were calculated by heat denaturation and determination of the amount of FAD released. ${ }^{22}$

\section{Steady-state kinetics}

Steady-state kinetic constants were measured by following spectrophotometrically the oxidation of $p$-methoxybenzyl alcohol into $p$-anisaldehyde $\left(\Delta \varepsilon_{285}=16,950 \mathrm{M}^{-1} \cdot \mathrm{cm}^{-1}\right)$. Bi-substrate kinetics were analysed by varying both the alcohol and $\mathrm{O}_{2}$ concentrations in $50 \mathrm{mM}$ sodium phosphate, $\mathrm{pH} 6.0$, at $10,15,20,25$ and $30{ }^{\circ} \mathrm{C}$. These reactions were performed in screw-cap cuvettes in which buffer was equilibrated using different $\mathrm{O}_{2} / \mathrm{N}_{2}$ mixtures that were bubbled for $15 \mathrm{~min}$ in a thermostatic bath at the desired temperatures. $\mathrm{O}_{2}$ solubility in water strongly depends on temperature and, thus, actual concentrations were calculated for each gas mixture and temperature. Reactions were triggered by addition of the alcohol substrate and AAO (3-5 nM final enzyme concentrations) with syringes into the cuvette with a final volume of $1 \mathrm{~mL}$. Initial rates were calculated as the ratio between absorbance and time from the linear phase of the oxidation of alcohol to aldehyde. Kinetic constants were obtained by fitting the observed rate constants to Eq. 1, describing a ternary mechanism:

$$
\frac{v}{\mathrm{e}}=\frac{k_{\mathrm{cat}} A B}{K_{\mathrm{m}(\mathrm{ox})} A+K_{\mathrm{m}(\mathrm{al})} B+K_{\mathrm{d}} B+A B}
$$

where $v$ stands for the initial velocity; $e$ is the enzyme concentration; $k_{\text {cat }}$ is the maximal turnover number; $\mathrm{A}$ is the alcohol concentration; $\mathrm{B}$ is the $\mathrm{O}_{2}$ concentration; and $K_{\mathrm{m}(\mathrm{ox})}$ and $K_{\mathrm{m}(\mathrm{al})}$ are the Michaelis-Menten constants for $\mathrm{O}_{2}$ and alcohol substrates, respectively.

\section{Rapid kinetics experiments}

Reduction rate constants were determined using a stopped-flow spectrophotometer from Applied Photophysics Ltd. (Surrey, United Kingdom), model SX17.MV, under anaerobic conditions. Solutions, buffers, substrates and enzymes (native AAO and mutated variants) were poured into glass tonometers, connected to an anaerobic train and subjected to 20-25 cycles of evacuation and Ar flushing. Glucose (10 mM final concentration) and glucose oxidase (10 U. $\mathrm{mL}$ $\left.{ }^{1}\right)$ were added after some vacuum-Ar cycles to all solutions to ensure anaerobiosis. The stopped-flow equipment was made anaerobic by flushing a solution of sodium dithionite that scavenged all traces of oxygen. Dithionite was then removed by rinsing the apparatus with anaerobic $50 \mathrm{mM}$ sodium phosphate, $\mathrm{pH}$ 
6.0. Reactions were followed with the photodiode array detector, and baseline was made with anaerobic $50 \mathrm{mM}$ sodium phosphate, $\mathrm{pH}$ 6.0. Kinetic constants were measured by mixing $\sim 20 \mu \mathrm{M}$ AAO with increasing concentrations of $p$-methoxybenzyl, $\left[\alpha-{ }^{2} \mathrm{H}_{2}\right]-p$ methoxybenzyl and $(R)-\left[\alpha-{ }^{2} \mathrm{H}\right]-p$-methoxybenzyl alcohols using the single-mixing mode at temperatures of: i) $6,8,10,12$ and $14{ }^{\circ} \mathrm{C}$ for native, Y92F and Y92L; and ii) $12,16,20$ and $25{ }^{\circ} \mathrm{C}$ for Y92W. Temperature was maintained constant with a thermostatic bath, and enzyme and substrate were equilibrated for $10 \mathrm{~min}$ prior to measurements. Observed rate constants $\left(k_{\mathrm{obs}}\right)$ were estimated by global fitting of the reduction spectra of the enzyme to a twospecies model using the ProKineticist software from Applied Photophysics. Reduction rate constants were estimated by nonlinear fitting of the observed rate constants at different substrate concentrations to Eq. 2:

$$
k_{\mathrm{obs}}=\frac{k_{\mathrm{red}} A}{K_{\mathrm{d}}+A}+k_{\mathrm{rev}}
$$

where $k_{\mathrm{obs}}$ is the observed rate for flavin reduction at a given substrate concentration (A); $k_{\text {red }}$ and $k_{\text {rev }}$ are the limiting rates for $\mathrm{HT}$ from the substrate to flavin N5 and its reverse reaction, respectively, at saturating substrate concentration; and $K_{\mathrm{d}}$ stands for the dissociation constant of the enzyme-substrate complex.

\section{Data analysis}

Data analysis was performed using SigmaPlot software (Systat Software Inc., San Jose, CA, USA). Temperature dependence of the calculated rate constants $\left(k_{\text {cat }}, k_{\text {cat }} / K_{\mathrm{m}}\right.$ and $\left.k_{\text {red }}\right)$ was estimated by fitting to the Arrhenius equation (Eq. 3). This allowed the estimation of the Arrhenius pre-exponential factors for protium and deuterium isotopes ( $A_{H}$ and $A_{D}$ respectively) and their corresponding activation energies $\left(E_{\mathrm{aH}}\right.$ and $\left.E_{\mathrm{aD}}\right)$. Primary KIE values $\left({ }^{\mathrm{D}} k\right.$ ) (i.e., the ratios of the rate with protium over the rates with deuterium) were determined using Eq. 4. Secondary KIE values $\left({ }^{D_{2}} k\right)$ were estimated as the ratio between the rate constants of mono-deuterated and di-deuterated substrates. Combination of Eqs. 3 and 4 led to the graphic representation of the temperature dependence of the KIE.

$$
\begin{aligned}
& k=A \times e^{\frac{E_{a}}{R T}} \\
& \mathrm{KIE}=\frac{k_{\mathrm{H}}}{k_{\mathrm{D}}}
\end{aligned}
$$

\section{Crystallization, data collection and AAO:p-anisic acid structure}

AAO was crystallized in complex with its final product and inhibitor $p$-anisic acid. Crystallization was achieved from a protein solution at $6 \mathrm{mg} \cdot \mathrm{mL}^{-1}$ mixed with $p$-anisic acid $(1.35 \mathrm{mM})$, both in $150 \mathrm{mM} \mathrm{NaCl}$ and $100 \mathrm{mM} \mathrm{NaK}_{2} \mathrm{PO}_{4}, \mathrm{pH}$ 7.0. $0.5 \mu \mathrm{L}$ of this mixture were added to $0.5 \mu \mathrm{L}$ of mother liquor consisting of $0.1 \mathrm{M}$ sodium acetate, $\mathrm{pH} 4.5$, and $1.0 \mathrm{M}$ di-ammonium hydrogen phosphate. Crystals were cryoprotected with reservoir solution containing $20 \%$ of glycerol. Diffraction data sets were collected on the 124 beamline at the Diamond Synchrotron (Oxfordshire, UK) at $100 \mathrm{~K}$ using a wavelength of $0.96862 \AA$. Data were processed, scaled and reduced with XDS $^{23}$ and SCALA ${ }^{24}$ from the CCP4 package. ${ }^{25}$ MOLREP ${ }^{26}$ from CCP4 was used to solve all the structures with the native AAO structure ( $\mathrm{pdb}$ 3FIM) as search model. Refinements were performed automatically by REFMAC $5^{27}$ from CCP4 and manually by $\mathrm{COOT}^{28}$. PROCHECK ${ }^{29}$ and MOLPROBITY ${ }^{30}$ were used to assess and validate the final structures.

Crystals belonged to the $\mathrm{P}_{4} 22$ hexagonal space group with one AAO: $p$-anisic acid complex in the asymmetric unit. Residues lacking electron density were not included in the final models. Statistics for data collection and refinement are shown in Table S1. Coordinates are deposited in the PDB with accession number 5001.

\section{Results}

\section{Crystal structure of AAO:p-anisic acid complex}

The crystal structure of AAO in complex with $p$-anisic acid was resolved at a resolution of $2.3 \AA$. $p$-Anisic acid acts as a competitive inhibitor of AAO that binds to the enzyme active site. ${ }^{11}$ It is also the product of the AAO reaction with the hydrated gem-diol form of $p$ anisaldehyde. ${ }^{13}$

The structural model of AAO: $p$-anisic acid complex comprises residues 2-566, 1 FAD, 1 -anisic acid, 5 glycerol and 280 water molecules. Structural superposition of the complex with the ligandfree AAO (pdb 3FIM) shows a root-mean-square deviation value of $0.21 \AA$ (superimposing $565 \mathrm{C} \alpha$ atoms) (Fig. 1A) concluding that their overall structures are pretty similar. The crystal structure shows the $p$-anisic acid tightly bound with distances from its carboxylic 0 atoms to the $\mathrm{N} \varepsilon$ atom of His502 (the catalytic base during the AAO reductive half reaction), the $\mathrm{N} \delta$ atom of His546 and the $\mathrm{N} 5$ atom of the FAD isoalloxazine ring of $2.55,2.72$ and $2.99 \AA$ respectively (Fig. 1B). This binding mode is compatible with redox catalysis, although in this structure it is the acid, instead of the alcohol, what is found as a ligand in the active site. Several other residues located at the active site, Phe397 and Tyr92, as well as the FAD itself, can establish hydrophobic interactions with atoms of the ligand molecule (Fig. 1B).

Tyr92, which is mutated in this work to unveil its involvement in the HT reaction, is located in a triad of residues - with Phe397 and Phe501- that acts as a hydrophobic bottleneck separating the active site of the enzyme from the outer environment. Moreover, it has been suggested that it establishes aromatic stacking interactions that help the substrate attain a catalytically competent position inside the active site. ${ }^{21}$ As depicted in Fig. 1B, the Tyr92 position does not seem altered upon the $p$-anisic acid binding, since it stabilises it through a T-stacking aromatic contact.

\section{Temperature dependence of AAO rate constants}

To investigate the temperature dependence of AAO catalysis, especially of HT during the reductive half reaction, bi-substrate steady-state and transient state kinetics were analysed for native AAO at different temperatures using deuterated forms of its physiological alcohol substrate. ${ }^{31} \alpha$-Protiated and two different $\alpha$ deuterated $p$-methoxybenzyl alcohols were employed: i) the monodeuterated substrate, in which only the pro- $R \mathrm{H}$ is substituted $\left((R)-\left[\alpha-{ }^{2} \mathrm{H}\right]-p\right.$-methoxybenzyl alcohol); and ii) its dideuterated counterpart, where both $\mathrm{H}$ atoms bound to the $\alpha$ carbon are isotopically substituted $\left(\left[\alpha-{ }^{2} \mathrm{H}_{2}\right]-p\right.$-methoxybenzyl alcohol). 

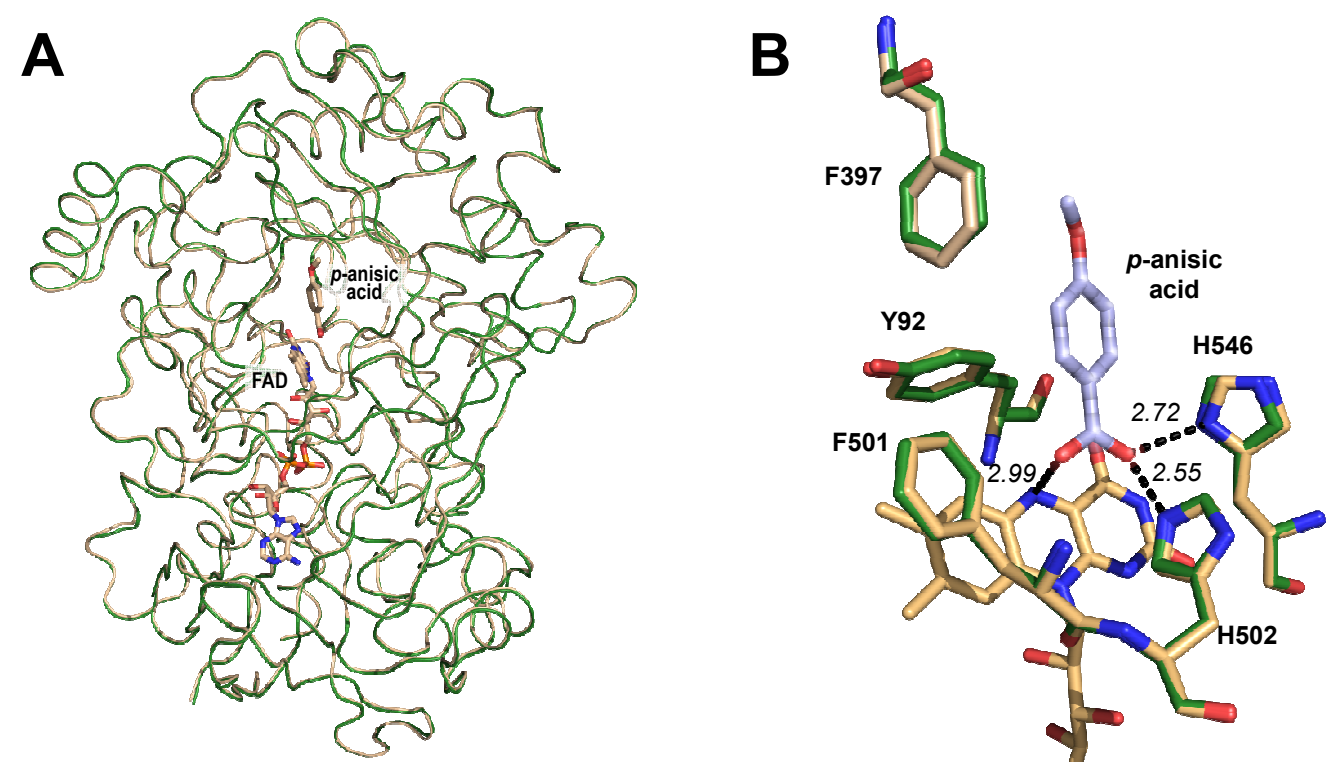

Fig. 1. Crystallographic structures of the AAO:p-anisic acid complex (pdb 5OC1, wheat) and ligand-free AAO (pdb 3FIM, green). A. Backbone superimposition. B. Detail of the superimposed active sites (CPK coloured, complex C atoms in wheat and free form C atoms in green) with distances (in $\AA$ ) from both carboxylic $\mathrm{O}$ to flavin $\mathrm{N} 5$ and $\mathrm{N} \varepsilon$ of His502.

Previous studies have described that AAO turnover $\left(k_{\mathrm{cat}}\right)$ is limited by the rate of $\mathrm{HT}$, reflecting only this chemical step. ${ }^{10}$ The temperature dependences of $k_{\text {cat }}$ values, together with the temperature dependence of the primary KIE values obtained, are shown in Fig. $2 \mathbf{A}$ and $\mathbf{B}$ (the kinetic constants estimated every $5^{\circ} \mathrm{C}$ between $10{ }^{\circ} \mathrm{C}$ and $30{ }^{\circ} \mathrm{C}$ for the $\alpha$-protiated and $\left[\alpha-{ }^{2} \mathrm{H}_{2}\right]-p$ methoxybenzyl alcohols are shown in Table S2). The turnover dependences were fitted to the Arrhenius equation to estimate the Arrhenius pre-exponential factors $(A)$ and the activation energy $\left(E_{a}\right)$, which allowed calculation of the differences in activation energies $\left(\Delta \mathrm{E}_{\mathrm{a}(\mathrm{D}-\mathrm{H})}=\mathrm{E}_{\mathrm{aD}}-\mathrm{E}_{\mathrm{aH}}\right)$ as well as the ratio between pre-exponential factors $\left(A_{H} / A_{D}\right)$ (Table 1 ). The $E_{a}$ for turnover with $\alpha$-deuterated substrate was higher than that obtained with the $\alpha$-protiated substrate, obtaining a large temperature-dependent $\mathrm{KIE}\left(\Delta \mathrm{E}_{\mathrm{a}(\mathrm{D}-\mathrm{H})}\right)$ and a value for the isotope effect on the Arrhenius pre-exponential factor below unity $\left(A_{\mathrm{H}} / A_{\mathrm{D}}<<1\right)$.

Table 1. Arrhenius parameters from the temperature dependence of the turnover $\left(k_{\text {cat }}\right)$ and hydride transfer $\left(k_{\text {red }}\right)$ reactions in the oxidation of $\alpha$-protiated and (di- and [R]mono-) $\alpha$-deuterated $p$ methoxybenzyl alcohols by native AAO.

\begin{tabular}{|c|c|c|c|}
\hline \multirow{5}{*}{$\begin{array}{l}\boldsymbol{A}_{\mathrm{H}}\left(\mathrm{s}^{-1}\right) \\
E_{\mathrm{aH}}\left(\mathrm{kcal} \cdot \mathrm{mol}^{-1}\right)\end{array}$} & \multirow{4}{*}{$\begin{array}{c}\boldsymbol{k}_{\text {cat }} \\
\alpha \text {-protiated } \\
9.8 \times 10^{6} \\
6.4\end{array}$} & \multirow{2}{*}{\multicolumn{2}{|c|}{$\begin{array}{c}\boldsymbol{k}_{\text {red }} \\
\alpha \text {-protiated }\end{array}$}} \\
\hline & & & \\
\hline & & \multicolumn{2}{|c|}{$2.5 \times 10^{8}$} \\
\hline & & \multicolumn{2}{|c|}{8.3} \\
\hline & {$\left[\alpha-{ }^{2} \mathrm{H}_{2}\right]$} & {$\left[\alpha-{ }^{2} \mathrm{H}_{2}\right]$} & $(R)-\left[\alpha-{ }^{2} H\right]$ \\
\hline$A_{\mathrm{D}}\left(\mathrm{s}^{-1}\right)$ & $2.3 \times 10^{13}$ & $3.6 \times 10^{8}$ & $4.2 \times 10^{10}$ \\
\hline$E_{\mathrm{aD}}\left(\mathrm{kcal} \cdot \mathrm{mol}^{-1}\right)$ & 16.2 & 9.8 & 12.2 \\
\hline$A_{\mathrm{H}} / A_{\mathrm{D}}$ & $4.3 \times 10^{-7}$ & 0.694 & 0.006 \\
\hline$\Delta E_{\mathrm{a}(\mathrm{D}-\mathrm{H})}\left(\mathrm{kcal} \cdot \mathrm{mol}^{-1}\right)$ & 9.9 & 1.5 & 3.9 \\
\hline
\end{tabular}

The high ${ }^{\mathrm{D}} k_{\text {cat }}$ values observed clearly indicated that the breakdown of $\mathrm{C} \alpha-\mathrm{H} / \mathrm{D}$ is limiting the flavin reduction step, and hence, the overall AAO catalysis. In contrast, the catalytic efficiency $\left(k_{\mathrm{cat}} / K_{\mathrm{m}(\mathrm{al})}\right)$ estimated using the $\alpha$-deuterated substrate showed a temperature dependence pattern similar to that for the $\alpha$-protiated substrate, leading to a KIE that is temperature independent (average $4.3 \pm 1.3$ ) (Fig. 2 C and D, and Table S2). This ${ }^{\mathrm{D}}\left(k_{\text {cat }} / K_{\mathrm{m}(\mathrm{all})}\right)$ value was lower than the ${ }^{\mathrm{D}} k_{\text {cat }}$ values (Table 2 ), indicating that the cleavage of $\mathrm{CH}$ bond becomes masked by other processes during overall AAO catalysis.

To reinforce the above results, the effect of temperature dependence on the rate constant for flavin reduction and the associate KIE effect were investigated to directly probe the HT of AAO $\left(k_{\text {red }}\right.$ and $K_{\mathrm{d}}$ values estimated with each substrate, every $2{ }^{\circ} \mathrm{C}$ between $6{ }^{\circ} \mathrm{C}$ and $14{ }^{\circ} \mathrm{C}$, are provided in Table S3). Arrhenius plots of the estimated $k_{\text {red }}$ for native AAO with each substrate are shown in Fig. 3A, and the thermodynamic parameters estimated by fitting to the Arrhenius equation are provided in Table 1 . The activation energy for $D^{-}$abstraction with $(R)-\left[\alpha-{ }^{2} \mathrm{H}\right]-p$-methoxybenzyl alcohol is higher than that obtained with $\left[\alpha-{ }^{2} \mathrm{H}_{2}\right]-p$-methoxybenzyl alcohol. As a consequence, the KIE estimated with the monodeuterated substrate is more temperature-dependent than that calculated for the dideuterated substrate $\left(3.9\right.$ and $1.5 \mathrm{kcal} \cdot \mathrm{mol}^{-1}$ for $\Delta \mathrm{E}_{\mathrm{a}(\mathrm{D}-\mathrm{H}) \text {, }}$ respectively), opposite to the pattern of primary ${ }^{\mathrm{D}} k_{\text {red }}$ (Table S4).

Table 2. KIEs determined from the temperature dependence of the steady-state kinetic parameters in native AAO reduction.

\begin{tabular}{ccc}
\hline $\mathbf{T}\left({ }^{\circ} \mathrm{C}\right)$ & ${ }^{\mathrm{D}} \boldsymbol{k}_{\text {cat }}$ & ${ }^{\mathrm{D}}\left(\boldsymbol{k}_{\text {cat }} / \boldsymbol{K}_{\mathbf{m}}\right)$ \\
\hline 10 & $15.6 \pm 0.6$ & $3.5 \pm 0.5$ \\
15 & $17.4 \pm 0.6$ & $4.9 \pm 0.6$ \\
20 & $9.1 \pm 0.2$ & $4.3 \pm 0.7$ \\
25 & $7.9 \pm 0.1$ & $3.9 \pm 0.2$ \\
30 & $5.4 \pm 0.4$ & $4.9 \pm 0.8$ \\
\hline
\end{tabular}

KIE values are the ratio between activities from bi-substrate kinetics on [ $\alpha$ $\left.\mathrm{H}_{2}\right] /\left[\alpha-{ }^{2} \mathrm{H}_{2}\right]$-alcohols (value \pm S.D.). 

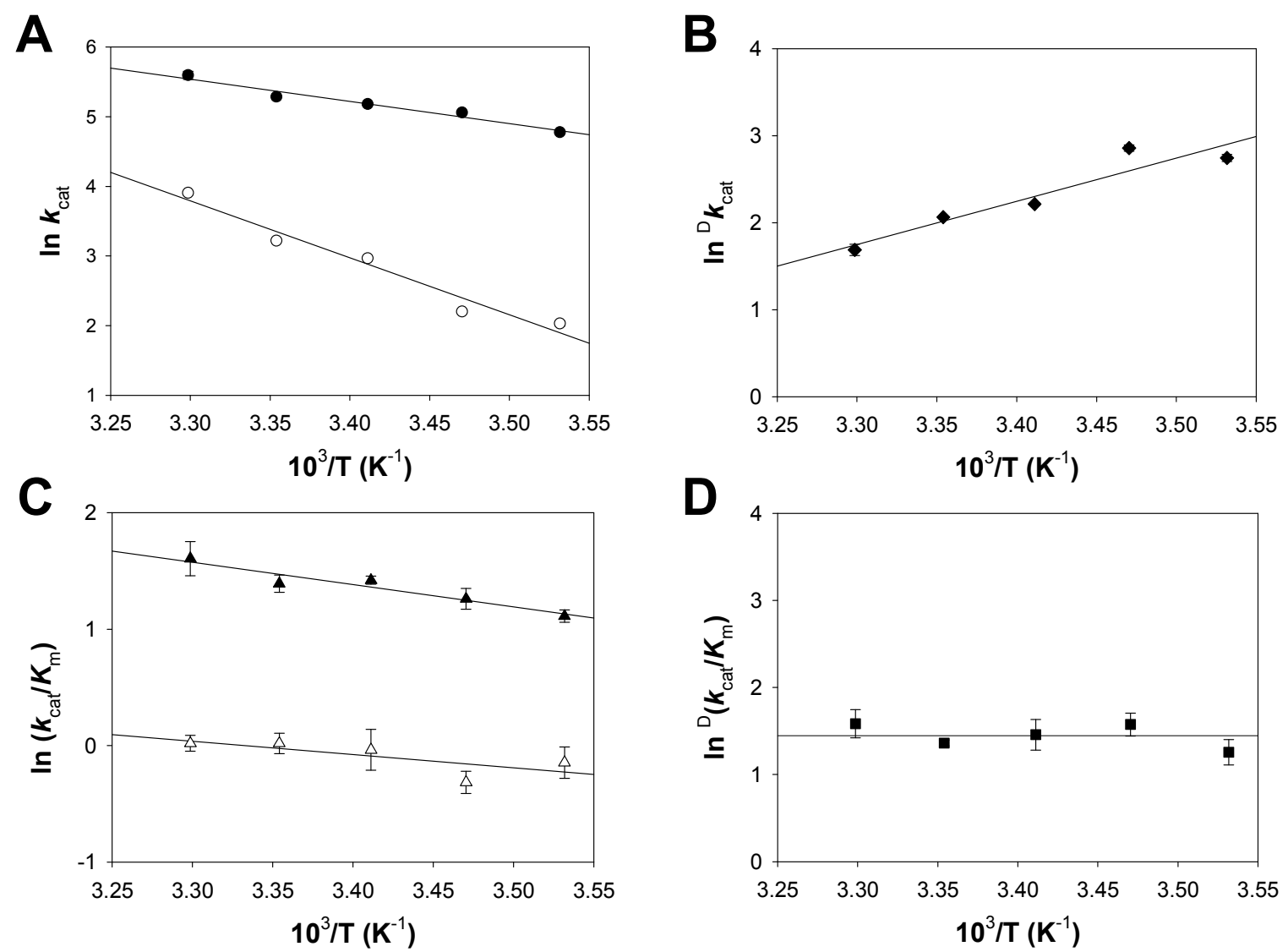

Fig. 2. Temperature dependences of steady-state kinetic parameters for native AAO reduction. A. Arrhenius plots of $k_{\text {cat }}$ with $\left[\alpha-\mathrm{H}_{2}\right]-p$-methoxybenzyl alcohol (filled circles) and [ $\left.\alpha-{ }^{2} \mathrm{H}_{2}\right]-p$-methoxybenzyl alcohol (open circles) B. Temperature dependence of the KIE of $k_{\text {cat }}\left({ }^{\mathrm{D}} k_{\text {cat }}\right)$. C. Arrhenius plots of $k_{\text {cat }} / K_{\mathrm{m}}$ with $\left[\alpha-\mathrm{H}_{2}\right]-p$-methoxybenzyl alcohol (filled triangles) and $\left[\alpha-{ }^{2} \mathrm{H}_{2}\right]-p$-methoxybenzyl alcohol (open triangles). D. Temperature dependence of the KIE of catalytic efficiency ${ }^{\mathrm{D}}\left(k_{\text {cat }} / K_{\mathrm{m}}\right)$. Vertical bars represent standard deviations. All data represented here are listed in Tables $\mathbf{2}$ and $\mathbf{S} \mathbf{2}$.

Besides, the values for $A_{H} / A_{D}$ were very close to zero and unity for $(R)-\left[\alpha-{ }^{2} \mathrm{H}\right]$ and $\left[\alpha-{ }^{2} \mathrm{H}_{2}\right]-p$-methoxybenzyl alcohols, respectively. The highest ${ }^{\mathrm{D}} k_{\text {red }}$ values obtained for the $\left[\alpha-{ }^{2} \mathrm{H}_{2}\right]-p$-methoxybenzyl alcohol with respect to those found for the $R$ isomer (Table 3 ) indicated a secondary KIE $\left({ }^{\mathrm{D}} k_{\text {red }}\right)$ that increases with temperature (Table S4), opposite to the behaviour of the primary ${ }^{D} k_{\text {red. }}$.

\section{Temperature dependence of the HT reaction in Tyr92 variants}

The role that the AAO binding pocket plays in HT modulation was investigated through the kinetic characterisation of the $\mathrm{HT}$ reaction of several Tyr92 variants (Y92F, Y92L and Y92W). The reductive half-

Table 3. KIEs determined from the temperature dependence of the $\mathrm{HT}$ reaction $\left(k_{\text {red }}\right)$ in native AAO reduction by (di- and [ $\left.R\right]$-mono-) $\alpha$ deuterated alcohols.

\begin{tabular}{ccc}
\hline $\mathbf{T}\left({ }^{\circ} \mathrm{C}\right)$ & {$\left[\boldsymbol{\alpha}^{2} \mathrm{H}_{2}\right]$} & $(\boldsymbol{R})-\left[\boldsymbol{\alpha}-{ }^{2} \mathrm{H}\right]$ \\
\hline 6 & $9.9 \pm 0.3$ & $6.7 \pm 0.3$ \\
8 & $9.9 \pm 0.1$ & $6.9 \pm 0.5$ \\
10 & $9.6 \pm 0.2$ & $6.0 \pm 0.3$ \\
12 & $9.1 \pm 0.2$ & $5.7 \pm 0.2$ \\
14 & $8.9 \pm 0.3$ & $5.8 \pm 0.3$ \\
\hline
\end{tabular}

$k_{\text {red }}$ constants were calculated for $\alpha$-protiated and $\alpha$-deuterated-[ $\left[\alpha-{ }^{2} \mathrm{H}_{2}\right]$ and $(R)-\left[\alpha-{ }^{2} \mathrm{H}\right]-p$-methoxybenzyl alcohols. KIE values for $k_{\text {red }}\left({ }^{D} k_{\text {red }}\right)$ are the ratio between HT constants on $\alpha$-protiated/ $\alpha$-deuterated alcohols (value \pm S.D.). reactions were analysed by using $\alpha$-protiated and $\left[\alpha-{ }^{2} \mathrm{H}_{2}\right]-p$ methoxybenzyl alcohols every $2{ }^{\circ} \mathrm{C}$ between $6{ }^{\circ} \mathrm{C}$ and $14{ }^{\circ} \mathrm{C}$ for $\mathrm{Y} 92 \mathrm{~F}$ and $\mathrm{Y} 92 \mathrm{~L}$, and at $12,16,20$ and $25^{\circ} \mathrm{C}$ for $\mathrm{Y} 92 \mathrm{~W}$. The spectral evolutions of these $Y 92$ variants with $\alpha$-deuterated substrate were similar to those previously reported with $\alpha$-protiated substrate, ${ }^{21}$ indicating a full two-electron flavin reduction (Fig. S1). The Y92F and Y92L variants showed similar reduction rates and affinity to those reported for native AAO, while Y92L showed 2-fold lower affinity ( $k_{\text {red }}$ and $K_{\mathrm{d}}$ values in Tables S5 and S6). Moreover, the incorporation of a bulkier residue in the $\mathrm{Y} 92 \mathrm{~W}$ variant produced a strong decrease in HT efficiency (7- and 200-fold lower reduction rate and affinity, respectively (Table S7).

Arrhenius plots for all Tyr92 variants are provided in Fig. 4, and their Arrhenius parameters are shown in Table 4. Regarding the Y92F variant, its overall behaviour resembles very closely that of the native AAO (Table 1), with similar activation energy for $\mathrm{D}^{-}$versus $\mathrm{H}^{-}$ abstraction. Therefore, their thermodynamic parameters were virtually identical to those calculated for the native $\left(\Delta \mathrm{E}_{\mathrm{a}(\mathrm{D}-\mathrm{H})} \approx 1.5\right.$ and $A_{H} / A_{D} \approx 0.6$ ).

Substitution of Tyr92 with leucine, a non-aromatic residue that probably produces some stacking interaction with the alcohol substrate, led to a slightly different behaviour. This variant has a sharpest temperature-dependent KIE than the native protein. The 

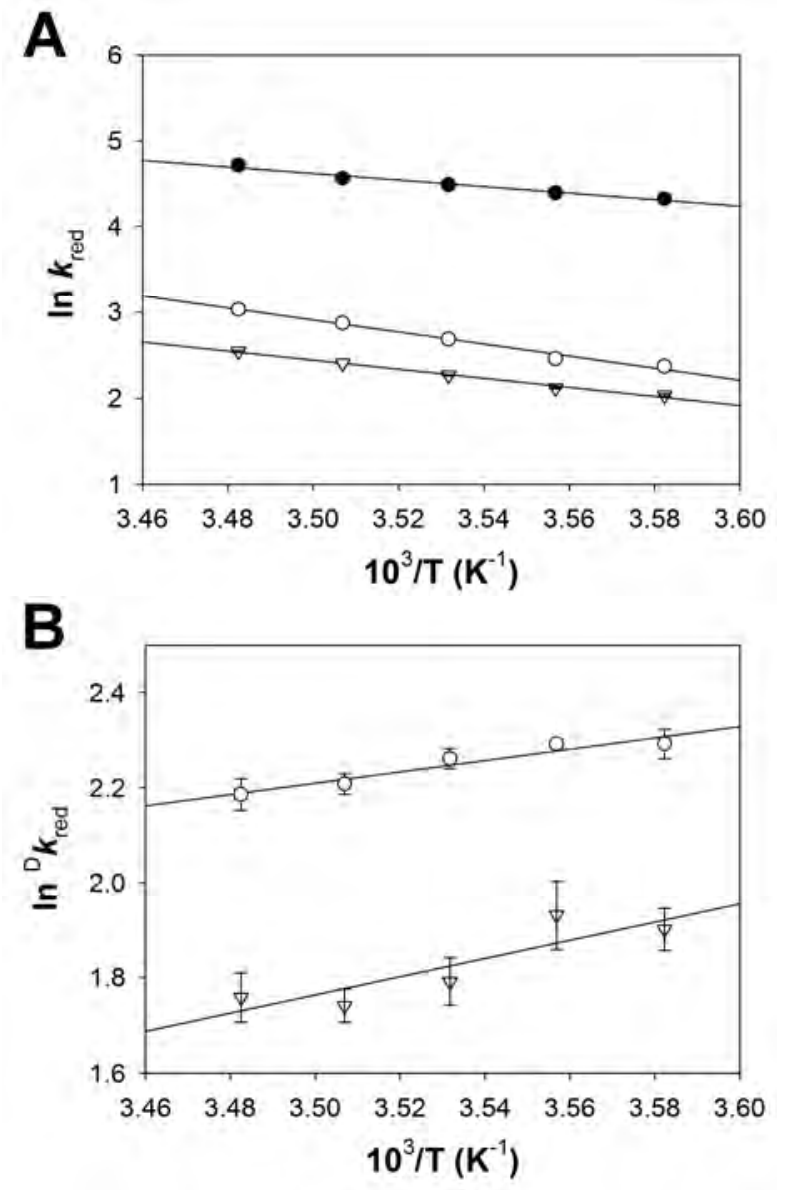

Table 4. Arrhenius parameters from the temperature dependence of the hydride transfer (HT) and deuteride transfer (DT) reaction $\left(k_{\text {red }}\right)$ in oxidation of $\left[\alpha-\mathrm{H}_{2}\right]$ and $(R)-\left[\alpha-{ }^{2} \mathrm{H}\right]-p$-methoxybenzyl alcohols by Tyr92 variants.

\begin{tabular}{|c|c|c|c|c|}
\hline & & Y92F & Y92L & Y92W \\
\hline & $\boldsymbol{k}_{\text {red }}\left(\mathrm{s}^{-1}\right)$ & $108.4 \pm 0.8$ & $90.1 \pm 0.4$ & $13.9 \pm 0.8$ \\
\hline HT & $\boldsymbol{A}_{\mathrm{H}}\left(\mathrm{s}^{-1}\right)$ & $3.1 \times 10^{8}$ & $9.7 \times 10^{6}$ & $7.7 \times 10^{7}$ \\
\hline & $E_{\mathrm{aH}}\left(\mathrm{kcal} \cdot \mathrm{mol}^{-1}\right)$ & 8.3 & 6.6 & 8.8 \\
\hline & $\boldsymbol{k}_{\text {red }}\left(\mathrm{s}^{-1}\right)$ & $12.3 \pm 0.1$ & $11.0 \pm 0.2$ & $1.5 \pm 0.1$ \\
\hline DT & $A_{\mathrm{D}}\left(\mathrm{s}^{-1}\right)$ & $4.8 \times 10^{8}$ & $3.4 \times 10^{9}$ & $1.5 \times 10^{7}$ \\
\hline & $E_{\mathrm{aD}}\left(\mathrm{kcal} \cdot \mathrm{mol}^{-1}\right)$ & 9.9 & 11.1 & 9.1 \\
\hline & $A_{\mathrm{H}} / A_{\mathrm{D}}$ & 0.591 & 0.003 & 5.260 \\
\hline & (D-H) $\left(\mathrm{kcal} \cdot \mathrm{mol}^{-1}\right)$ & 1.6 & 4.5 & 0.3 \\
\hline
\end{tabular}

Measurements in $50 \mathrm{mM}$ phosphate, $\mathrm{pH}$, every $2^{\circ} \mathrm{C}$ between 6 and $14^{\circ} \mathrm{C}$ for $\mathrm{Y} 92 \mathrm{~F}$ and $\mathrm{Y} 92 \mathrm{~L}$ and at $12,16,20$ and $25^{\circ} \mathrm{C}$ for $\mathrm{Y} 92 \mathrm{~W}$. $k_{\text {red }}$ constants at different temperatures were fitted to the Arrhenius equation to obtain the parameters shown above.

Therefore, the catalytic N5 of FAD is only accessible through a hydrophobic funnel-shaped channel with a bottleneck delimited by lateral side chains of Tyr92, Phe397 and Phe501. Diffusion simulations of the $p$-methoxybenzyl alcohol substrate into the AAO active site described motion reorganization of the Gln395-Thr406 loop, including Phe397 side chain oscillations to allow the entrance of substrates. ${ }^{14}$ Once in the active site, the alcohol substrate would adopt a catalytically competent position suitable for a concerted proton abstraction from its $\alpha$-hydroxyl by the His 502 catalytic base and $H T$ from the pro- $R$ hydrogen to the flavin N5 at a distance of 2.4-2.5 $\AA$.

In this work, the AAO crystal structure complexed with $p$-anisic

Fig. 3. Temperature dependences of the $H T$ reaction for the reduction of native AAO A. Arrhenius plots of $k_{\text {red }}$ with $\left[\alpha-\mathrm{H}_{2}\right]-p$-methoxybenzyl alcohol (filled circles), $(R)-\left[\alpha-{ }^{2} \mathrm{H}\right]-p$-methoxybenzyl alcohol (open circles) and $\left[\alpha-{ }^{2} \mathrm{H}_{2}\right]-$ $p$-methoxybenzyl alcohol (triangles). B. Temperature dependence of the KIE of $k_{\text {red }}\left({ }^{D} k_{\text {red }}\right)$ with $(R)-\left[\alpha-{ }^{2} \mathrm{H}\right]-p$-methoxybenzyl alcohol (triangles) and $\left[\alpha-{ }^{2} \mathrm{H}_{2}\right]-$ $p$-methoxybenzyl alcohol (open circles). Vertical bars represent standard deviations. All data represented here are listed in Tables $\mathbf{3}$ and $\mathbf{S 3}$.

Y92L variant showed more temperature-dependent rates for $\mathrm{D}^{-}$ abstraction than for $\mathrm{H}^{-}$abstraction $\left(\triangle \mathrm{E}_{\mathrm{a}(\mathrm{D}-\mathrm{H})}\right.$ is larger than that for native $A A O)$. As a consequence, the $Y 92 \mathrm{~L}$ variant isotope effect on the Arrhenius pre-exponential factor is very close to zero.

Substitution of Tyr92 with tryptophan seems to disrupt the behaviours analysed so far with $p$-methoxybenzyl and $\left[\alpha-{ }^{2} \mathrm{H}_{2}\right]-p$ methoxybenzyl alcohols. The activation energy for $D^{-}$abstraction by the $\mathrm{Y} 92 \mathrm{~W}$ variant shows a temperature dependence that is similar to that for $\mathrm{H}^{-}$abstraction $\left(\triangle \mathrm{E}_{\mathrm{a}(\mathrm{D}-\mathrm{H})}\right.$ is below unity), leading to a virtually temperature independent $\mathrm{KIE}$ and a $A_{\mathrm{H}} / A_{\mathrm{D}}$ value greater than unity.

\section{Discussion}

\section{AAO structure fosters $\mathrm{HT}$}

Unlike other crystal structures from the GMC superfamily, the structure of $P$. eryngii AAO shows a buried active site with a unique GIn395-Thr406 loop that restricts the access of substrates. ${ }^{8}$ acid closely resembles that of the previously theoretical model of AAO:p-methoxybenzyl alcohol complex with the $\mathrm{C} \alpha$ atom of ligand and the FAD N5 atom at a distance of $2.8 \AA$ (Fig. S3). ${ }^{14}$ Remarkably, the AAO active-site cavity is already pre-formed to attain the catalytically competent geometry and does not undergo any conformational changes upon inhibitor binding. Moreover, overall comparison of this complex structure with the ligand-free AAO structure shows minimal rearrangements at the fold level, which cannot explain how alcohol substrate is completely embedded inside an inaccessible active site.

Burial of substrates is a common feature developed by a large number of flavooxidases involved in HT reactions. ${ }^{32-35}$ Some of them, as D-amino acid oxidase and cholesterol oxidase, show open and closed conformations with active-site gates controlling the entrance of ligands into the biding pocket. Others, such as the vanillyl-alcohol oxidase (VAO) involved in the oxidation of phenolic benzyl alcohols, do not show any apparent structural elements involved in such conformational changes and bind ligands without modifying the conformation of its catalytic residues. ${ }^{36}$ Thus, AAO and VAO show a highly pre-organized active site able to oxidize a broad range of benzyl alcohols. 

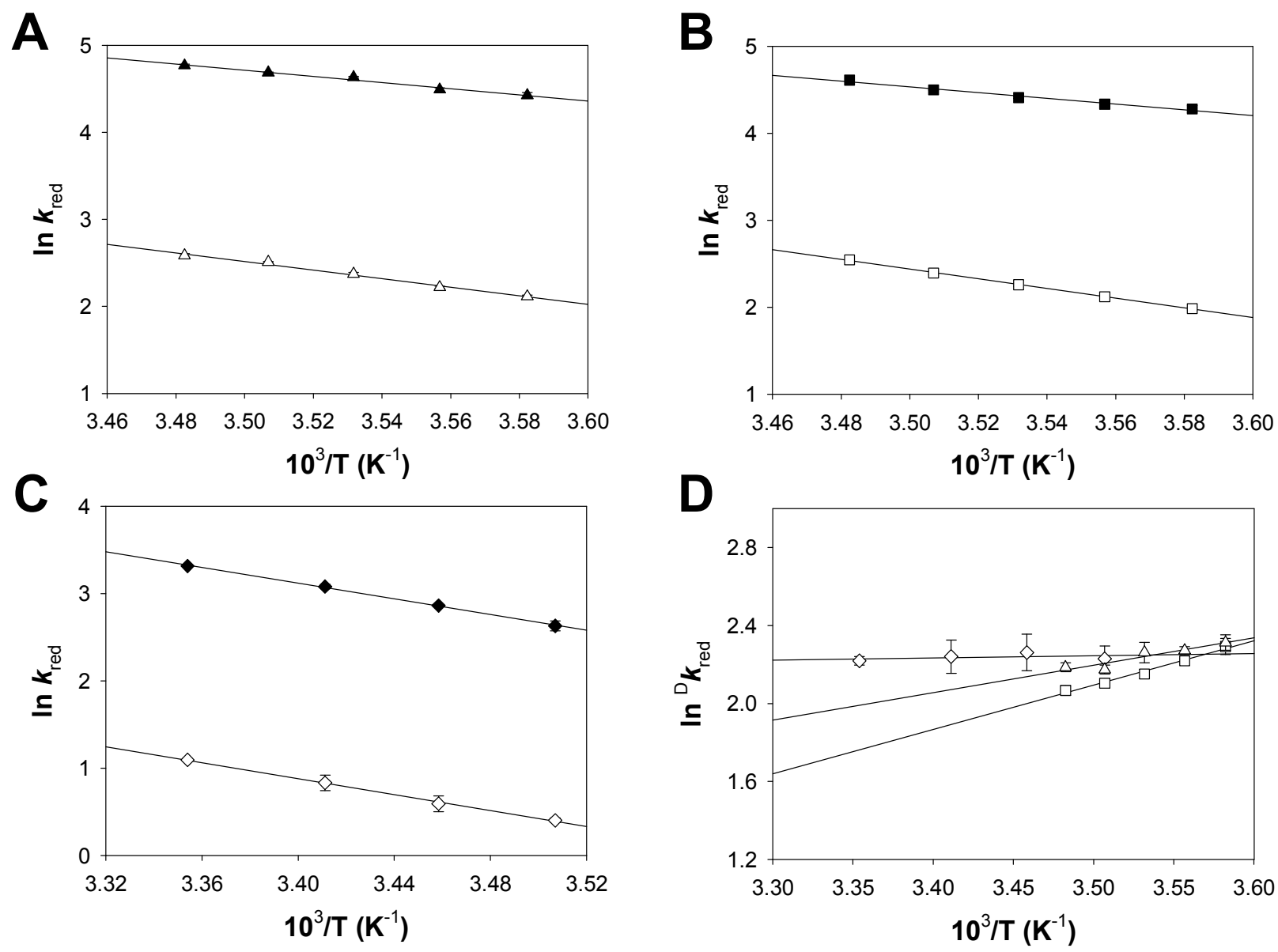

Fig. 4. Temperature dependences of the HT reaction for the reduction of AAO Y92 variants: A. Arrhenius plots for the reduction of Y92F with $p$ methoxybenzyl alcohol (filled triangles) and $\left[\alpha-{ }^{2} \mathrm{H}_{2}\right]-p$-methoxybenzyl alcohol (open triangles). B. Arrhenius plots for the reduction of $Y 92 \mathrm{~L}$ with [ $\alpha-$ $\mathrm{H}_{2}$ ]-p-methoxybenzyl alcohol (filled squares) and $\left[\alpha-{ }^{2} \mathrm{H}_{2}\right]-p$-methoxybenzyl alcohol (open squares). $\mathrm{C}$. Arrhenius plots for the reduction of $\mathrm{Y} 92 \mathrm{~W}$ with $\left[\alpha-\mathrm{H}_{2}\right]-p$-methoxybenzyl alcohol (filled diamonds) and $\left[\alpha-{ }^{2} \mathrm{H}_{2}\right]-p$-methoxybenzyl alcohol (open diamonds). D. Temperature dependence of ${ }^{\mathrm{D}} k_{\text {red }}$ for Y92F (triangles), Y92L (squares) and Y92W (diamonds). Vertical bars represent standard deviations. All data presented here are listed in Tables 4 and S5-S7.

\section{Environmentally-modulated tunnelling in AAO HT reaction}

We also studied the effect of temperature on the KIEs with deuterated $p$-methoxybenzyl alcohol to further investigate whether HT proceeds through tunnelling in AAO. Previous studies indicated that the AAO overall turnover is limited by the $\mathrm{H}^{-}$or $\mathrm{D}^{-}$transfer from $p$-methoxybenzyl alcohol to the N5 of FAD. ${ }^{10}$ The obtained temperature effects on the $k_{\text {cat }}$ and ${ }^{\mathrm{D}} k_{\text {cat }}$ values suggest that tunnelling plays a role in $\mathrm{HT}$ during AAO catalysis. Evidence for this assumption is provided by the temperature-dependence of ${ }^{\mathrm{D}} k_{\text {cat }}$ with values higher than expected in a semiclassical approach, as well as with the $A_{H} / A_{\mathrm{D}}$ ratio very close to zero. However, the lower ${ }^{\mathrm{D}}\left(k_{\text {cat }} / K_{\text {m(al) }}\right)$ values when compared to the ${ }^{\mathrm{D}} k_{\text {cat }}$ and ${ }^{\mathrm{D}} k_{\text {red }}$ indicated kinetic complexity for $p$-methoxybenzyl alcohol oxidation. In the catalytic mechanism of $\mathrm{AAO}$, the ${ }^{\mathrm{D}}\left(k_{\text {cat }} / K_{\mathrm{m}(\mathrm{al})}\right)$ value is given as follows:

$$
{ }^{D}\left(\frac{k_{c a t}}{K_{m(a l)}}\right)=\frac{{ }^{D} k_{2}+C_{f}+{ }^{D} E q C_{r}}{1+C_{f}+C_{r}}
$$

where, ${ }^{\mathrm{D}} k_{2} \sim{ }^{\mathrm{D}} k_{\text {red, }}$ is the intrinsic isotope effect for the cleavage of the $p$-methoxybenzyl alcohol $\mathrm{CH}$ bond, $C_{f}$ and $C_{r}$ are the forward and reverse commitments to catalysis on catalytic efficiency, respectively; and ${ }^{D} E q$ is the equilibrium isotope effect, which has a value of 1.24 for the conversion of an alcohol to an aldehyde. ${ }^{37}$

The irreversibility of the AAO HT process and the lack of effect of oxygen concentration on the ${ }^{\mathrm{D}}\left(k_{\text {cat }} / K_{\mathrm{m}(\mathrm{al})}\right)$ (KIE $\sim 4$ between $0.05-$ $1.3 \mathrm{mM} \mathrm{O}_{2}$ at $20{ }^{\circ} \mathrm{C}$ ) are consistent with a negligible reverse commitment to catalysis. As a consequence, any decrease in the observed ${ }^{\mathrm{D}}\left(k_{\mathrm{cat}} / K_{\mathrm{m}(\mathrm{al})}\right)$ must be due to the forward commitment to catalysis, which is given by the ratio of the rate constant for the HT step on the rate constant for the dissociation of enzyme:substrate complex $\left(k_{2} / k_{-1}\right)$.

To avoid this kinetic complexity, the involvement of tunnelling in the HT reaction was directly investigated on the AAO reductivehalf reaction by transient kinetic assays. In this regard, the temperature dependence of ${ }^{D} k_{\text {red }}$ with $p$-methoxybenzyl alcohol and $(R)-\left[\alpha-{ }^{2} \mathrm{H}\right]-p$-methoxybenzyl alcohol agrees well with the steadystate parameters, suggesting that tunnelling plays a role in the HT. 
Moreover, the $A_{H} / A_{\mathrm{D}}$ value very close to zero and the more favourable HT compared to DT (large value for $\Delta \mathrm{E}_{\mathrm{a}(\mathrm{D}-\mathrm{H})}$ with protium than with deuterium) indicate that DAD sampling is critical to reach the optimal tunnelling conformation. This gating model agrees well with the $p$-anisic binding observed in our AAO complexed structure, where, by similarity the reducing substrate is predicted to bind in a catalysis-ready conformation. It is worth mentioning that a combination of passive and active dynamics tunnelling is observed when using $\left[\alpha^{-}{ }^{2} \mathrm{H}_{2}\right]-p$-methoxybenzyl alcohol $\left(A_{H} / A_{D} \sim 1\right)$. The ${ }^{{ }^{D}} k_{\text {red }}$ observed reflects a contribution of the vibrations of the pro-S hydrogen to those of the pro- $R$ hydrogen at the ground state and the tunnelling ready state during HT with $p$-methoxybenzyl alcohol. Thus, the gating contribution is the one already observed with $(R)$ $\left[\alpha-{ }^{2} \mathrm{H}\right]-p$-methoxybenzyl alcohol. On the other hand, the passive component is due to the secondary isotope effects expressing isotopic differences in zero point energy that change along the reaction coordinate of the motion assisted-activated hydrogen tunnelling model (appearing as isotopic differences in the reorganisation energy and the reaction driving force). ${ }^{38-40}$

\section{Tyr92 contributes to environmentally-modulated HT in AAO}

The crystal structure of the AAO: $p$-anisic complex agrees very well with previous studies indicating that the stacking-stabilising interaction of the aromatic substrate with the Tyr92 active site residue is essential for $\mathrm{HT}$ in alcohol oxidation by AAO. ${ }^{21}$ Therefore, disruption of these interactions could have even deleterious effects on AAO active site configuration and HT process. In this study, the contribution of this residue to the alcohol oxidation has been evidenced using the Y92F, Y92L and Y92W AAO variants.

Replacement of Tyr92 with phenylalanine did not show any significant difference in HT efficiency with regard to native AAO. Analogously the effects on the temperature dependence for $\mathrm{HT}$ and DT with $\left[\alpha-\mathrm{H}_{2}\right]$ and $\left[\alpha-{ }^{2} \mathrm{H}_{2}\right]-p$-methoxybenzyl alcohols were similar to those observed for the native protein, suggesting that gating is the main force acting during tunnelling.

The Y92L and Y92W variants showed, in contrast, critical changes from the behaviour of the native enzyme. In this regard, the reduced isotope effect on $A_{H} / A_{D}$ observed with Y92L together with its higher temperature dependence for DT indicate that DAD sampling is more relevant in this variant than in native AAO. Such an increase in gating contribution suggests that the $Y 92 \mathrm{~L}$ active site is more flexible than that of the native AAO. Moreover, Y92L compromises the optimal disposition of the reacting atoms, as deduced from its higher $K_{\mathrm{d}}$ and lower HT efficiency, what can be compensated for increasing the DAD sampling. Similar results were reported for soybean lipoxygenase upon reduction in size of the side chain of an active-site residue. ${ }^{41}$

Regarding the Y92W mutant, the introduction of a bulkier residue increases the rigidity of the active site, thereby impairing the gating contribution to catalysis. This assumption is a consequence of the temperature-independent ${ }^{\mathrm{D}} k_{\text {red }}$ and the large $A_{H} / A_{D}$ ratio. These results do not indicate, however, that the mutation improves the configuration of the active site by eliminating the need for the gating contribution during catalysis, since HT efficiency for this variant is reduced by almost 100 -fold with regard to native AAO. Therefore, the introduction of this tryptophan in the AAO active site might result in a different stacking interaction with the alcohol substrate at a less favourable position for $\mathrm{HT}^{21}$, thus hampering its efficient orientation for gating. Consequently, the variant is forced to rely on passive dynamics to attain a tunnelling relevant position for $\mathrm{HT}$, which could be the reason why catalysis is impaired.

\section{Conclusions}

Results herein reported point towards HT taking place through quantum-mechanical tunnelling in AAO as deduced from the temperature dependence of the reaction parameters. Fast active protein motions, also known as gating, proved to play a key role in the HT from substrate to the FAD cofactor of the enzyme. Structural data reinforce the hypothesis by offering insight into the distances over which the particle is transferred. Structural and kinetic data suggest that the AAO active site is highly pre-organised to accommodate the substrate in a catalytically relevant position that only requires thermally-activated DAD sampling for the HT. Motions within the substrate molecule proved to be essential for DAD sampling, as suggested by the secondary KIE using differentlydeuterated substrates, and the different behaviour in the reduction of the enzyme with these substrates. Disruption of the AAO active site structure, by replacement of a residue directly involved in substrate positioning into the active site, shows that the enzyme commitment to catalysis is changed and other protein motions, i.e. passive dynamics, might be involved in compensating for this alteration.

\section{Acknowledgements}

The authors thank Pedro Merino (University of Zaragoza) for synthesising the deuterated alcohol substrates, and Jorge Estrada (Barcelona Supercomputing Center) for discussion and helpful suggestions. This work was supported by the European INDOX (KBBE-2013-7-613549) and EnzOx2 (H2020-BBI-PPP-2015-720297) projects, and the NOESIS (BIO2014-56388-R) and FLADIMOTEC (BIO2016-75183-P) projects of the Spanish Ministry of Economy and Competitiveness. J.C. acknowledges an FPU fellowship (FPU20122041) from the Spanish Ministry of Education, Culture and Sports.

\section{References}

1. J. Basran, L. Masgrau, M. J. Sutcliffe, and N. S. Scrutton. in A. Kohen and H.-H. Limbach (eds.), Isotope effects in chemistry and biology. CRC Press, Boca Raton. 2006, pp. 671-689.

2. A. Hernández-Ortega, P. Ferreira and A. T. Martínez, Appl. Microbiol. Biotechnol., 2012, 93, 1395-1410.

3. D. Floudas, M. Binder, R. Riley, K. Barry, R. A. Blanchette, B. Henrissat, A. T. Martínez, R. Otillar, J. W. Spatafora, J. S. Yadav, A. Aerts, I. Benoit, A. Boyd, A. Carlson, A. Copeland, P. M. Coutinho, R. P. de Vries, P. Ferreira, K. Findley, B. Foster, J. Gaskell, D. Glotzer, P. Górecki, J. Heitman, C. Hesse, C. Hori, K. Igarashi, J. A. Jurgens, N. Kallen, P. Kersten, A. Kohler, U. Kües, T. K. A. Kumar, A. Kuo, K. LaButti, L. F. Larrondo, E. Lindquist, A. Ling, V. Lombard, S. Lucas, 
T. Lundell, R. Martin, D. J. McLaughlin, I. Morgenstern, E. Morin, C. Murat, M. Nolan, R. A. Ohm, A. Patyshakuliyeva, A. Rokas, F. J. Ruiz-Dueñas, G. Sabat, A. Salamov, M. Samejima, J. Schmutz, J. C. Slot, F. St.John, J. Stenlid, H. Sun, S. Sun, K. Syed, A. Tsang, A. Wiebenga, D. Young, A. Pisabarro, D. C. Eastwood, F. Martin, D. Cullen, I. V. Grigoriev and D. S. Hibbett, Science, 2012, 336, 1715-1719.

4. P. Ferreira, J. Carro, A. Serrano and A. T. Martínez, Mycologia, 2015, 107, 1105-1119.

5. J. Carro, A. Serrano, P. Ferreira, and A. T. Martínez. in V. K. Gupta and M. G. Tuohy (eds.), Microbial Enzymes in Bioconversions of Biomass. Springer, Berlin, Germany. 2016, pp. 301-322.

6. J. Carro, P. Ferreira, L. Rodríguez, A. Prieto, A. Serrano, B. Balcells, A. Ardá, J. Jiménez-Barbero, A. Gutiérrez, R. Ullrich, M. Hofrichter and A. T. Martínez, FEBS J., 2015, 282, 3218-3229.

7. A. Hernández-Ortega, P. Ferreira, P. Merino, M. Medina, V. Guallar and A. T. Martínez, ChemBioChem, 2012, 13, 427-435.

8. I. S. Fernández, F. J. Ruiz-Dueñas, E. Santillana, P. Ferreira, M. J. Martínez, A. T. Martínez and A. Romero, Acta Crystallogr. D. Biol. Crystallogr., 2009, 65, 1196-1205.

9. A. Hernández-Ortega, F. Lucas, P. Ferreira, M. Medina, V. Guallar and A. T. Martínez, Biochemistry, 2012, 51, 6595-6608.

10. P. Ferreira, A. Hernández-Ortega, B. Herguedas, A. T. Martínez and M. Medina, J. Biol. Chem., 2009, 284, 24840-24847.

11. P. Ferreira, M. Medina, F. Guillén, M. J. Martínez, W. J. H. van Berkel and A. T. Martínez, Biochem. J., 2005, 389, 731-738.

12. F. Guillén, A. T. Martínez and M. J. Martínez, Eur. J. Biochem., 1992, 209, 603-611.

13. P. Ferreira, A. Hernández-Ortega, B. Herguedas, J. Rencoret, A. Gutiérrez, M. J. Martínez, J. JiménezBarbero, M. Medina and A. T. Martínez, Biochem. J., 2010, 425, 585-593.

14. A. Hernández-Ortega, K. Borrelli, P. Ferreira, M. Medina, A. T. Martínez and V. Guallar, Biochem. J., 2011, 436, 341-350.

15. Z. D. Nagel and J. P. Klinman, Nat. Chem. Biol., 2009, 5, 543-550.

16. L. Y. P. Luk, E. J. Loveridge and R. K. Allemann, Phys. Chem. Chem. Phys., 2015, 17, 30817-30827.

17. R. K. Allemann, R. M. Evans, L. H. Tey, G. Maglia, J. Pang, R. Rodriguez, P. J. Shrimpton and R. S. Swanwick, Philos Trans R Soc Lond B Biol Sci, 2006, 361, 1317.

18. F. Fan and G. Gadda, J. Am. Chem. Soc., 2005, 127, 17954-17961.

19. J. P. Klinman, Acc. Chem. Res., 2015, 48, 449-456.

20. P. Singh, K. Francis and A. Kohen, ACS Catal., 2015, 5, 3067-3073.
21. P. Ferreira, A. Hernández-Ortega, K. Borrelli, F. Lucas, B. Herguedas, V. Guallar, A. T. Martínez and M. Medina, FEBS J., 2015, 282, 3091-3106.

22. F. J. Ruiz-Dueñas, P. Ferreira, M. J. Martínez and A. T. Martínez, Protein Express. Purif., 2006, 45, 191-199.

23. W. Kabsch, Acta Crystallographica Section DBiological Crystallography, 2010, 66, 125-132.

24. W. Kabsch, J. Appl. Crystallogr., 1988, 21, 916-924.

25. N. 4. Collaborative Computational Project, Acta Crystallogr. D. Biol. Crystallogr., 1994, 50, 760-763.

26. A. Vagin and A. Teplyakov, J. Appl. Crystallogr., 1997, 30, 1022-1025.

27. G. N. Murshudov, A. A. Vagin and Dodson D.J., Acta Crystallogr. D. Biol. Crystallogr., 1997, 53, 255.

28. P. Emsley, B. Lohkamp, W. G. Scott and K. Cowtan, Acta Crystallographica Section D-Biological Crystallography, 2010, 66, 486-501.

29. R. A. Laskowski, M. W. Macarthur, D. S. Moss and J. M. Thornton, J. Appl. Crystallogr., 1993, 26, 283-291.

30. V. B. Chen, W. B. Arendall, J. J. Headd, D. A. Keedy, R. M. Immormino, G. J. Kapral, L. W. Murray, J. S. Richardson and D. C. Richardson, Acta Crystallographica Section D-Biological Crystallography, 2010, 66, 12-21.

31. A. Gutiérrez, L. Caramelo, A. Prieto, M. J. Martínez and A. T. Martínez, Appl. Environ. Microbiol., 1994, 60, 1783-1788.

32. J. Li, A. Vrielink, P. Brick and D. M. Blow, Biochemistry, 1993, 32, 11507-11515.

33. J. P. Kim, M. Wang and R. Paschke, Proc. Natl. Acad. Sci. USA, 1993, 90, 7523-7527.

34. P. Rowland, F. S. Nielsen, K. F. Jensen and S. Larsen, Structure, 1997, 5, 239-252.

35. A. Mattevi, M. A. Vanoni, F. Todone, M. Rizzi, A. Teplyakov, A. Coda, M. Bolognesi and B. Curti, Proc. Natl. Acad. Sci. USA, 1996, 93, 7496-7501.

36. A. Mattevi, M. W. Fraaije, A. Mozzarelli, L. Olivi, A. Coda and W. J. H. van Berkel, Structure, 1997, 5, 907 920.

37. W. W. Cleland, Methods Enzymol., 1980, 64, 104-125.

38. M. Derakhshani-Molayousefi, S. Kashefolgheta, J. E. Eilers and Y. Lu, J. Phys. Chem. A, 2016, 120, 4277 4284.

39. D. Roston, Z. Islam and A. Kohen, Arch. Biochem. Biophys., 2014, 544, 96-104.

40. A. Kohen, Acc. Chem. Res., 2015, 48, 466-473.

41. M. P. Meyer, D. R. Tomchick and J. P. Klinman, Proc. Natl. Acad. Sci. U. S A, 2008, 105, 1146-1151. 